\title{
Abstract from Current Literature
}

Incidence and Prevalence of Childhood Epilepsy: A Nationwide Cohort Study

Kari Modalsli Aaberg, Nina Gunnes, Inger Johanne Bakken, Camilla Lund Søraas, Aleksander Berntsen, Per Magnus, Morten I. Lossius, Camilla Stoltenberg, Richard Chin, Pål Surén.

Pediatrics. May 2017, VOLUME 139.

Background and Objectives: Epilepsy affects $0.5 \%$ to $1 \%$ of children and is the most frequent abstract chronic neurologic condition in childhood. Incidence rates appear to be declining in highincome countries. The validity of epilepsy diagnoses from different data sources varies, and contemporary population-based incidence studies are needed.

Methods: The study was based on the Norwegian Mother and Child Cohort Study. Potential epilepsy cases were identified through registry linkages and parental questionnaires. Cases were validated through medical record reviews and telephone interviews of parents.

Results: The study population included 112, 744 children aged 3 to 13 years (mean 7.4 years) at end of registry follow-up (December 31, 2012). Of these, 896 had registry recordings and/or questionnaire reports of epilepsy. After validation, 587 (66\%) met the criteria for an epilepsy diagnosis. The incidence rate of epilepsy was 144 per 100000 person-years in the first year of life and 58 per 100000 for ages 1 to 10 years. The cumulative incidence of epilepsy was $0.66 \%$ at age 10 years, with $0.62 \%$ having active epilepsy. The 309 children (34\%) with erroneous reports of epilepsy from the registry and/or the questionnaires had mostly been evaluated for nonepileptic paroxysmal events, or they had undergone electroencephalography examinations because of other developmental or neurocognitive difficulties.

Conclusions: Approximately 1 out of 150 children is diagnosed with epilepsy during the first 10 years of life, with the highest incidence rate observed during infancy. Validation of epilepsy diagnoses in administrative data and cohort studies is crucial because reported diagnoses may not meet diagnostic criteria for epilepsy.
Vitamin D supplementation to prevent acute respiratory tract infections: systematic review and meta-analysis of individual participant data

Adrian R Martineau, David A Jollif, Richard L Hooper, Lauren Greenberg, John F Aloia, , Peter Bergman, Gal Dubnov-Raz, Susanna Esposito, Davaasambuu Ganmaa, Adit A Ginde, Emma C Goodall, Cameron C Grant, Christopher J Griffiths, Wim Janssens, llkka Laaksi, Semira Manaseki-Holland, , David Mauger, David R Murdoch.

British Medical Journal, 15 $5^{\text {th }}$ February, 2017.

Objectives To assess the overall effect of vitamin D supplementation on risk of acute respiratory tract infection, and to identify factors modifying this effect.

Design Systematic review and meta-analysis of individual participant data (IPD) from randomised controlled trials.

Data sources Medline, Embase, the Cochrane Central Register of Controlled Trials, Web of Science, ClinicalTrials.gov, and the International Standard Randomised Controlled Trials Number registry from inception to December 2015.

Eligibility criteria for study selection Randomised, double blind, placebo controlled trials of supplementation with vitamin $D_{3}$ or vitamin $D_{2}$ of any duration were eligible for inclusion if they had been approved by a research ethics committee and if data on incidence of acute respiratory tract infection were collected prospectively and prespecified as an efficacy outcome.

Results 25 eligible randomised controlled trials (total 11321 participants, aged 0 to 95 years) were identified. IPD were obtained for 10933 (96.6\%) participants. Vitamin D supplementation reduced the risk of acute respiratory tract infection among all participants (adjusted odds ratio $0.88,95 \%$ confidence interval 0.81 to 0.96 ; $\mathrm{P}$ for heterogeneity $<0.001$ ). In subgroup analysis, protective effects were seen in those receiving daily or weekly vitamin $D$ without additional bolus doses (adjusted odds ratio 0.81, 0.72 to 0.91 ) but not in those receiving one or more bolus doses (adjusted odds ratio $0.97,0.86$ to $1.10 ; \mathrm{P}$ for 
interaction $=0.05$ ). Among those receiving daily or weekly vitamin $\mathrm{D}$, protective effects were stronger in those with baseline 25-hydroxyvitamin D levels $<25$ $\mathrm{nmol} / \mathrm{L}$ (adjusted odds ratio $0.30,0.17$ to 0.53 ) than in those with baseline 25-hydroxyvitamin D levels e"25 $\mathrm{nmol} / \mathrm{L}$ (adjusted odds ratio $0.75,0.60$ to 0.95 ; $P$ for interaction=0.006). Vitamin $D$ did not influence the proportion of participants experiencing at least one serious adverse event (adjusted odds ratio 0.98, 0.80 to $1.20, P=0.83$ ). The body of evidence contributing to these analyses was assessed as being of high quality.

Conclusions Vitamin D supplementation was safe and it protected against acute respiratory tract infection overall. Patients who were very vitamin $D$ deficient and those not receiving bolus doses experienced the most benefit are at an impasse. In such situations, the instrumental role played by a child's deterioration and avoidance of the courts risks giving insufficient weight to the child's interests. New approaches to decision making are needed.

\section{Best interests' in paediatric intensive care: an empirical ethics study}

Giles Birchley, Rachael Gooberman, Zuzana Deans, James Fraser, Richard Huxtable.

Archives of Disease in Childhood. April 13, 2017

Objective In English paediatric practice, English law requires that parents and clinicians agree the "best interests' of children and, if this is not possible, that the courts decide. Court intervention is rare and the concept of best interests is ambiguous. We report qualitative research exploring how the best interests standard operates in practice, particularly with decisions related to planned non-treatment. We discuss results in the light of accounts of best interests in the medical ethics literature.

Design We conducted 39 qualitative interviews, exploring decision making in the paediatric intensive care unit, with doctors, nurses, clinical ethics committee members and parents whose children had a range of health outcomes. Interviews were audiorecorded and analysed thematically.

Results Parents and clinicians indicated differences in their approaches to deciding the child's best interests. These were reconciled when parents responded positively to clinicians' efforts to help parents agree with the clinicians' view of the child's best interests. Notably, protracted disagreements about a child's best interests in non-treatment decisions were resolved when parents' views were affected by witnessing their child's physical deterioration. Negotiation was the norm and clinicians believed avoiding the courts was desirable.

Conclusions Sensitivity to the long-term interests of parents of children with life-limiting conditions is defensible but must be exercised proportionately. Current approaches emphasise negotiation but offer few alternatives when decisions. 